and

$$
\begin{aligned}
& S(\alpha, \gamma, x)=(-1)^{\gamma \Gamma} \Gamma(\alpha-\gamma+1) \Gamma(\gamma-1) \frac{\Gamma(\gamma)}{\Gamma(\alpha)} x^{1-\gamma} \\
& \times\left(1+\frac{\alpha-\gamma+1}{2-\gamma} \frac{x}{1 !}+\frac{(\alpha-\gamma+1)(\alpha-\gamma+2)}{(2-\gamma)(3-\gamma)} \frac{x^{2}}{2 !}+\cdots\right. \\
&\quad \text { to } \gamma-1 \text { terms }) \\
&= \frac{\gamma-1}{\alpha-1} \frac{1}{x}-\frac{(\gamma-1)(\gamma-2)}{(\alpha-1)(\alpha-2)} \frac{1 !}{x^{2}}+\frac{(\gamma-1)(\gamma-2)(\gamma-3)}{(\alpha-1)(\alpha-2)(\alpha-3)} \frac{2 !}{x^{3}}-\cdots \\
& \quad+(-1)^{\gamma} \frac{(\gamma-1)(\gamma-2) \cdots 2.1}{(\alpha-1)(\alpha-2) \cdots(\alpha-\gamma+1)} \frac{(\gamma-2) !}{x^{\gamma-1}} .
\end{aligned}
$$

This function $S(\alpha, \gamma, x)$ is the part omitted by Webb and Airey, and others. Of course, if $\gamma=1$, then $S=0$ and the solution given by Webb and Airey is correct.

$$
\text { J. C. P. Miller }
$$

The University Mathematical Laboratory

Cambridge, England

1. $A$ Webb \& Airey-Adams-Bateman-Olsson Error, M.T.E. 117, MTAC, v. 2, 1947, p. 352-353.

2. A. Fletcher, J. C. P. Miller, \& L. Rosenhead, An Index of Mathematical Tables, Scientific Computing Service Ltd., London, 1946, article 22.56, p. 336.

\title{
Approximation and Table of the Weierstrass $\&$ Function in the Equianharmonic Case for Real Argument
}

The most readily accessible table of the Weierstrass $\&$ function for the equianharmonic case appears to be that in Jahnke-Emde [2], which is mainly to 4D.

The equianharmonic case for the function $\wp\left(u ; g_{2}, g_{3}\right)$ is that in which $g_{2}=0$, $g_{3}=1$. The function behaves like $1 / u^{2}$ near the origin. This leads us to consider tabulating

$$
f(u ; 0,1) \equiv \wp(u ; 0,1)-1 / u^{2}
$$

Because of symmetries, we may restrict attention to the interval $0<u \leq \omega_{2}$, where $\omega_{2} \approx 1.52995$ and is the real half-period of $\varnothing$; see F. Tricomi [1].

It is possible to represent $\varnothing$ on the above interval to $7 \mathrm{~S}$ by a relatively simple approximant. Write

$$
f(u ; 0,1) \approx c_{3} u^{4}+c_{6} u^{10}+c_{9} u^{16}+c_{12} u^{22}+c_{16} u^{28},
$$

the polynomial being obtained by truncation of Maclaurin's series. Thus we have

$$
\wp(u ; 0,1) \approx g(u) / u^{2}=h(y) / u^{2}
$$


where $g(u)=1+u^{2} f(u ; 0,1), y=u^{6}$, and $h(y)$ is of the fifth degree. It turns out that $h$ can be "economized by the use of Chebyshev polynomials" in the sense of C. Lanczos [3] so that 7S values of $\wp$ can be obtained on the interval of interest using a cubic approximation to $h(y)$. That is

(4) $\varphi(u ; 0,1) \approx H(y) / u^{2}$, where $y=u^{6}$ and $H(y)=$

$$
\begin{array}{rl}
.99999 & 990 \\
+.035714504 y \\
+.00009803869 y^{2} \\
+.00000019320 y^{3} .
\end{array}
$$

At the half-period $u=\omega_{2} \approx 1.52995, H(y) / u^{2} \approx 0.62996055$ whereas the true value for $\varphi\left(\omega_{2}\right)=4^{-1} \approx 0.62996052$.

A compact printed table of the auxiliary function $f$ has been constructed for

\begin{tabular}{|c|c|c|c|c|c|}
\hline$u$ & $f(u ; 0,1)$ & $\delta^{2 *} f$ & $u$ & $f(u ; 0,1)$ & $\delta^{2 *} f$ \\
\hline 0.0 & .0000000 & 85 & 0.80 & .0146391 & 6889 \\
\hline 0.1 & .0000036 & $+\quad 341$ & 0.85 & .0186624 & 7795 \\
\hline 0.2 & .0000571 & 1630 & 0.90 & .0234664 & 8766 \\
\hline 0.3 & .0002893 & $37 \quad 70$ & 0.95 & .0291483 & 9810 \\
\hline 0.4 & .0009143 & 6771 & 1.00 & .0358126 & 10927 \\
\hline 0.5 & .0022322 & 10632 & 1.05 & $.04357 \quad 12$ & 12130 \\
\hline 0.6 & .0046292 & 15353 & 1.10 & .0525446 & 13432 \\
\hline 0.7 & $.00857 \quad 78$ & 20957 & 1.15 & $.06286 \quad 32$ & $148 \quad 39$ \\
\hline 0.8 & .0146391 & $274 \quad 77$ & 1.20 & .0746681 & 16377 \\
\hline & & & 1.25 & $.08811 \quad 35$ & 18064 \\
\hline & & & 1.30 & .1033686 & 19930 \\
\hline & & & 1.35 & .1206207 & 22011 \\
\hline & & & 1.40 & .1400787 & 24353 \\
\hline & & & 1.45 & $.16197 \quad 78$ & 27008 \\
\hline & & & 1.50 & 1865849 & 30052 \\
\hline & & & 1.55 & .2142060 & 33570 \\
\hline
\end{tabular}
use with Everett's Interpolation Formula to second (modified) differences. It follows. The differences have been modified by throwback of fourth differences

Table of Weierstrass Function $f=\varnothing-1 / u^{2}$.

using the throwback constant -0.18393 . This throwback process is described by various authors; see for example, the NBS work [4] or F. B. Hildebrand [5].

The formula for $H(y)$ has been published previously without comment (see Review 58, p. 110 this issue $M T A C)$.

Thomas H. SouthaRd

University of California

Los Angeles, California

This paper was prepared under the sponsorship of the Office of Naval Research.

1. F. TRICOMI, Elliptische Funktionen, Akademische Verlagsgesellschaft, Leipzig, 1948, p. 20.

2. E. JAHNKE \& F. EMdE, Tables of Functions with Formulae and Curves, 4th ed., Dover Publications, New York, 1945, p. 100.

3. NBS Applied Mathematics Series No. 9, Tables of Chebyshev Polynomials $S_{n}(x)$ and $C_{n}(x)$, U. S. Gov. Printing Office, Washington, D. C., 1952, p. 15.

4. NBSCL, Tables of Bessel Functions of Fractional Order, v. 1, Columbia University Press, New York, 1948, p. 33.

5. F. B. HILDEBRAND, Introduction to Numerical Analysis, McGraw-Hill Book Co., Inc., New York, 1956, p. 112. 